\title{
Long-Term White Matter Changes after Severe Traumatic Brain Injury: A 5-Year Prospective Cohort
}

\author{
J. Dinkel, A. Drier, O. Khalilzadeh, V. Perlbarg, V. Czernecki, R. Gupta, F. Gomas, P. Sanchez, D. Dormont, D. Galanaud, R.D. Stevens, and \\ L. Puybasset for NICER (Neuro Imaging for Coma Emergence and Recovery) Consortium
}

$\therefore \mathrm{O}-\mathrm{EBM}_{2}$

\begin{abstract}
BACKGROUND AND PURPOSE: Extensive white matter damage has been documented in patients with severe traumatic brain injury, yet how this damage evolves in the long term is not well understood. We used DTI to study white matter changes at 5 years after traumatic brain injury.
\end{abstract}

MATERIALS AND METHODS: There were 8 healthy control participants and 13 patients with severe traumatic brain injury who were enrolled in a prospective observational study, which included clinical assessment and brain MR imaging in the acute setting ( $<6$ weeks) and 2 years and 5 years after injury. Only subjects with mild to moderate disability or no disability at 1 year were included in this analysis. DTI parameters were measured in 20 different brain regions and were normalized to values obtained in an age-matched control group.

RESULTS: In the acute setting, fractional anisotropy was significantly lower in the genu and body of the corpus callosum and in the bilateral corona radiata in patients compared with control participants, whereas radial diffusivity was significantly $(P<.05)$ higher in these tracts. At 2 years, fractional anisotropy in these tracts had further decreased and radial diffusivity had increased. No significant changes were detected between 2 and 5 years after injury. The baseline radial diffusivity and fractional anisotropy values in the anterior aspect of the brain stem, genu and body of the corpus callosum, and the right and left corona radiata were significantly $(P<.05)$ associated with neurocognitive sequelae (including amnesia, aphasia, and dyspraxia) at year 5.

CONCLUSIONS: DTI changes in major white matter tracts persist up to 5 years after severe traumatic brain injury and are most pronounced in the corpus callosum and corona radiata. Limited structural change is noted in the interval between 2 and 5 years.

ABBREVIATIONS: $\mathrm{FA}=$ fractional anisotropy; $\mathrm{L} 1=$ axial diffusivity; $\mathrm{Lt}=$ radial diffusivity; $\mathrm{MD}=$ mean diffusivity; $\mathrm{TBI}=$ traumatic brain injury

$\mathbf{T}$ raumatic brain injury (TBI) represents a significant public health burden around the globe and is an important cause of death and lifelong disability. ${ }^{1}$ Severe TBI accounts for only $10 \%$ of

Received January 4, 2013; accepted after revision March 20.

From the Department of Radiology (J.D., O.K., R.G.), Massachusetts General Hospital, Harvard Medical School, Boston, Massachusetts; Department of Radiology (J.D.), University Hospital Heidelberg, Heidelberg, Germany; Departments of Neuroradiology (A.D., D.D., D.G.), Neurology (V.C.), and Anesthesiology and Intensive Care (P.S., L.P.), Pitié Salpêtrière Hospital, Assistance Publique-Hôpitaux de Paris, France; INSERM, UMRS 678 (V.P.) and INSERM, CRICM UMR S975 (V.C.), Université Pierre et Marie Curie-Paris 6, Paris, France; Department of Anesthesiology and Intensive Care (F.G.), Louis Mourier Hospital, Colombes, France; and Division of Neuroscience Critical Care (R.D.S.), Department of Anesthesiology Critical Care Medicine, Johns Hopkins University School of Medicine, Baltimore, Maryland.

This study was funded by a grant from the French Ministry of Health (Projet Hospitalier de Recherche Clinique registration \#P051061 [2005]) and from departmental funds from the Assistance Publique-Hôpitaux de Paris.

Please address correspondence to Pr. Louis Puybasset, Neuro-réanimation Chirurgicale Babinski, Département d'Anesthésie-Réanimation, Groupe Hospitalier PitiéSalpêtrière, Université Pierre et Marie Curie, Paris 6, 47-83, Bd de l'hôpital; 75013 Paris, France; e-mail: louis.puybasset@psl.aphp.fr; louis.puybasset@me.com

- Indicates open access to non-subscribers at www.ajnr.org

Evidence-Based Medicine Level 2.

http://dx.doi.org/10.3174/ajnr.A3616 these injuries, but it involves an extended stay in intensive care units and may cause subsequent long-term functional disability that needs long-term inpatient and outpatient rehabilitation. ${ }^{2}$

Beside focal lesions, moderate and severe TBI is associated with diffuse axonal injuries that manifest with little or no apparent damage in conventional neuroimaging but can be seen postmortem on microscopy studies. ${ }^{3}$ Diffuse axonal injury can lead to a disconnection of critical cortical and subcortical pathways and might represent a neural basis for the cognitive dysfunction seen in the long term after TBI. ${ }^{3}$ Because conventional diagnostic imaging fails to appreciate the burden of microstructural white matter damage that occurs in TBI, it has limited ability to predict long-term outcome in a broad range of cognitive behavioral and functional impairments. DTI is an MR imaging technique that measures the properties of water diffusion. ${ }^{4,5}$ The degree of diffusion is characterized by 3 eigenvectors representing the diffusion in each orthogonal direction. Diffusion in white matter is particularly limited and anisotropic because of physical obstacles to water diffusion imposed by the myelin and axon membranes. ${ }^{6,7}$ The diffusion properties are believed to reflect 
many factors, including myelination and axonal attenuation and integrity. ${ }^{6,7}$

DTI has shown promising results for depiction of neuropathologic changes in TBI, in which the microarchitecture of white matter may be altered. Although previous studies have shown the association of changes in DTI parameters of the white matter with different types of TBI (mild, moderate, or severe; in acute or chronic phase) and its outcome, ${ }^{6-15}$ limited evidence is available on the pattern of long-term changes in patients with chronic severe TBI. Studies have suggested evidence of active brain remodeling and neuroplasticity ${ }^{16}$ after TBI in children. ${ }^{17}$ However, the long-term white matter modifications in adults with TBI need to be further characterized. Knowledge of the time course and extent of these changes might yield better approaches for therapy and rehabilitation. ${ }^{18}$ Here, we undertook a prospective longitudinal study to determine the nature of white matter changes in a subset of patients with severe TBI who had moderate or no gross disability 1 year after injury by using DTI imaging. We hypothesized that microstructural cerebral alterations identified within the first weeks after the severe TBI would persist 2 and 5 years after injury.

\section{MATERIALS AND METHODS}

The institutional review board of Pitié Salpêtrière Hospital approved this study protocol. Informed consent was obtained from all study participants (legally authorized representative of the patients during the acute stage, patients themselves after recovery of consciousness, and healthy volunteers who served as control participants).

\section{Patients}

Patients with severe TBI (Glasgow Coma Scale, 3-8; posttraumatic amnesia $>7$ days; and loss of consciousness $>24$ hours $^{1}$ ) who were admitted to the intensive care unit were enrolled in this prospective study between March 2005 and March 2008. Inclusion criteria were 1 ) adult patients ( $\geq 18$ years) and 2 ) moderate or no disability at 1 year (Glasgow Outcome Scale at 4 or 5). Exclusion criteria were 1) moribund patients (expected survival $<24$ hours); 2) physiologic instability (eg, from hemodynamic instability, increased intracranial pressure, and/or rapidly deteriorating respiratory function) that would preclude MR imaging; 3 ) contraindication to the MR imaging; 4) penetrating head injury; and 5) a central nervous system condition such as stroke, brain tumor, or a neurodegenerative disease preceding TBI.

\section{Control Participants}

Eight age- and sex-matched healthy volunteers $(n=8$; mean age, $32 \pm 8$ years; age range, $22-49$ years; men) were enrolled in this study. They did not have a history of TBI or neurologic disease.

\section{Clinical Data Collection}

We collected data by using standardized case report forms including demographics, Glasgow Coma Scale, and cranial CT scan results. Head CT scans were performed within 48 hours after admission to the intensive care unit. A central study monitoring was performed to ensure data accuracy. The Glasgow Outcome Scale was assessed for all patients at 1 year post TBI. Baseline MR imaging was acquired as soon as clinically feasible (within 6 weeks after TBI). The MR imaging scans were repeated at 2 and 5 years after the TBI.
At 5 years, patients underwent a comprehensive neuropsychological evaluation. The level of disability on the basis of the Glasgow Outcome Scale, the disability rating scale, the extended Glasgow Outcome Score, and the modified Rankin Scale were assessed, the results of which will be reported separately. For the purposes of this study, patients were classified according to whether they had moderate cognitive sequelae (including amnesia, aphasia, and dyspraxia).

\section{MR Imaging}

Control participants underwent MR imaging by use of the same acquisition protocol as in the patients and underwent subsequent imaging after 2 years. MR imaging was performed on a $1.5 \mathrm{~T}$ system (Signa; GE Healthcare, Milwaukee, Wisconsin) with the participants under sedation and mechanical ventilation for the first MR imaging and without any sedation for the follow-up MR imaging scans. The acquisition protocol included morphologic sequences (FLAIR, T2*-weighted gradient-echo, T2-weighted turbo spin-echo, T1-weighted 3D inversion recovery fast-spoiled gradient recalled), MR spectroscopy, and DTI. Only DTI results are presented in this study. DTI was performed by use of the following parameters: axial plane; 1 volume at b-value, $0 \mathrm{~s} / \mathrm{mm}^{2}$; 24 directions; diffusion b-value, $700 \mathrm{~s} / \mathrm{mm}^{2}$; section thickness, 5 $\mathrm{mm}$; no gap; 20 sections; field of view, $32 \times 32 \mathrm{~cm}$; matrix size, $128 \times 128$; and 2 averages. The inferior section of the DTI acquisition was positioned at the junction between the pons and the medulla oblongata. Additional details on the DTI acquisition protocol are available in our prior study. ${ }^{19}$

\section{MR Imaging Analysis}

DTI images were preprocessed by use of FSL software (http:// www.fmrib.ox.ac.uk/fsl). ${ }^{20}$ The local diffusion parameters, namely, fractional anisotropy (FA), mean diffusivity (MD), axial diffusivity (L1), and radial diffusivity (Lt), were calculated ${ }^{21}$ for the entire brain in each patient and control participant. To make diffusion measures comparable between patients and participants, the FA, MD, L1, and Lt maps were registered on a $1 \times 1 \times$ $1 \mathrm{~mm}^{3}$ standard space image (Montreal Neurological Institute 152 space) by use of the tract-based spatial statistics procedure. ${ }^{22}$ Correction for distortions from eddy currents was performed by use of the $b=0$ images. The whole brain, including the pathologic regions, was registered by use of an automated nonrigid technique, and individual FA, MD, L1, and Lt values were projected on an alignment-invariant template for the brain. This procedure maps all available information to a common brain template and avoids misalignment among participants. A total of 20 ROIs for DTI analysis were selected, based on the ICBM-DTI-81 white matter atlas. ${ }^{23}$ This atlas consists of 48 white matter tracts. Because some of these ROIs are very small and are subject to partial volume effects, we merged the initial 48 white matter tracts into 20 larger regions as published previously ${ }^{19,24}$ (in posterior fossa: 1middle cerebellar peduncle; 2 - anterior brain stem; 3 - posterior brain stem; 4- right cerebral peduncle; 5 - left cerebral peduncle; in deep brain: 6- genu of the corpus callosum; 7- body of the corpus callosum; 8- splenium of the corpus callosum; 9- right side anterior arm of the internal capsule; 10- left side anterior arm of the internal capsule; 11- right side posterior arm of the internal cap- 
sule; 12 - left side posterior arm of the internal capsule; in superficial brain regions: 13- right stratum sagittale; 14- left stratum sagittale; 15- right superior longitudinal fasciculus; 16- left superior longitudinal fasciculus; 17- right external capsule; 18- left external capsule; 19 - right corona radiata; 20 - left corona radiata). The regional DTI parameter extraction consisted of 3 steps: a nonlinear registration of the FA map to a template (provided by FSL), a projection of FA onto the FA template skeleton representing the centers of all tracts (also provided by FSL), and averaging of FA measures within the 20 ROIs restricted to the skeleton. The ROIs are placed automatically after nonrigid registration on a defined position on the common brain template. Furthermore, the registered maps were visually verified and were compared with the template. In total, the average values of FA, MD, L1, and Lt in these 20 ROIs resulted in 80 DTI biomarkers for each participant.

During the 5 years needed for the study, our MR scanner underwent 2 software updates. The sequence parameters were kept identical except for the TE, which had to be slightly modified (initial TE, $81 \mathrm{~ms}$; then $85.9 \mathrm{~ms}$; and finally $84.3 \mathrm{~ms}$ ). To account for TE-related variability in raw DTI values and to make our results are comparable with future studies, we normalized the raw DTI parameter values for each participant. For this purpose, every time our scanner undergoes a software, material, or sequence update, we scan several healthy participants for DTI metric normalization. We have constituted a very large dataset of reference values for our scanner that we use routinely for normalization in our clinical workflow and in previous studies. ${ }^{19,24}$ For this study, the raw FA, MD, L1, and Lt values in each of the 20 preselected ROIs of both patients and control participants were divided by the mean values of the corresponding reference group. Further details of MR image processing and normalization are provided in a previous publication. ${ }^{19}$

\section{Statistical Analysis}

We performed the statistical analysis by using SPSS software (version 16.0; IBM, Armonk, New York). The required sample size to detect a significant association at $\alpha=0.05$ and with a power of $80 \%$ was estimated to be 15 . Continuous variables are expressed as mean \pm standard deviation. Variables had normal distribution (according to the Kolmogorov-Smirnov test); therefore, parametric tests were used. One-way repeated-measures ANOVA was used to compare different DTI values between baseline and the follow-up steps, in patients with severe TBI. $P$ values were corrected for multiple testing ${ }^{25}$ (Bonferroni method), and only the corrected $P$ values are presented in the study. One-way ANOVA (with Bonferroni post hoc) was used to compare baseline DTI values between healthy control participants and patients with TBI with and without neurocognitive sequelae. An independent sample $t$ test was used to compare changes (year 2 vs baseline) in DTI values between patients and control participants. The level of significance was set at $P<.05$.

\section{RESULTS}

A total of 15 patients met the inclusion criteria during the study period. Two patients were dropped because of unwillingness to undergo the follow-up scans. There was no death or loss to follow-up in the remaining patients. The analysis was done in the 13

\section{Patient characteristics}

\begin{tabular}{lcc}
\hline & & Range \\
\hline Age (y) & $32.4(9.0)$ & $(18-49)$ \\
Men/women, $n$ & $13 / 0$ & - \\
GCS at admission, $n$ (SD) & $6(4)$ & $(3-8)$ \\
ICU stay duration (d) & $47.5(23.1)$ & $(10-97)$ \\
Assisted ventilation duration (d) & $33.1(16.9)$ & $(9-72)$ \\
Type of accident, $n$ (\%) & & \\
$\quad$ MVA & $10(77)$ & - \\
$\quad$ Assault, fall, other & $3(23)$ & - \\
Hematoma, $n(\%)^{\mathrm{a}}$ & & \\
$\quad$ Epidural & $3(23)$ & - \\
Subdural & $1(8)$ & - \\
$\quad$ Subarachnoid hemorrhage & $8(61)$ & - \\
Midline shift & $3(23)$ & - \\
Compressed third ventricle & $4(31)$ & - \\
Contusion, $n$ (\%) & $10(77)$ & - \\
Neurosurgical intervention, $n(\%)$ & $2(15)$ & - \\
Neurologic sequelae, $n$ (\%) & & - \\
Amnesia & $2(15)$ & - \\
Aphasia & $1(8)$ & \\
Dyspraxia & $2(15)$ & - \\
\hline
\end{tabular}

Note:-Variables are presented as mean (standard deviation) or number (percentage).

GCS indicates Glasgow Coma Scale; ICU, intensive care unit; SD, standard deviation; MVA, motor-vehicle collision.

${ }^{a}$ Hematoma was defined as an area spontaneously hyperdense on the first CT scan. ${ }^{b}$ Contusion was defined as a focal area appearing hypodense or of mixed density on the CT scan.

patients with severe TBI (Table 1) and in 8 control participants. There was no significant difference in age between patients and healthy control participants (patients: mean age, $32 \pm 9$ years; age range, $18-49$ years; control participants: mean age, $32 \pm 8$ years; age range, $22-49$ years). Initial (baseline) scan was obtained at a mean of $22 \pm 12$ days (range, 12-58 days) after injury. Repeated scans were performed at a mean of 2.0 years (range, $1.8-2.1$ years) and 5.0 years (range, $4.7-5.4$ years) after TBI. The mean rescan time for healthy control participants was $2.0 \pm 0.08$ years. The automatic segmentation software accurately recognized the 20 ROIs in all patients, even in the presence of intracranial pathologic features. A typical set of FLAIR and FA images of a patient is shown in Fig 1.

For healthy control participants, there was no difference in white matter measurable by DTI between baseline and year 2. In patients with severe TBI at baseline, FA values were significantly lower at the genu and body of the corpus callosum and the bilateral corona radiata compared with control participants (Fig 2), whereas Lt was significantly higher in the same regions. Repeatedmeasures ANOVA in patients with TBI (comparing baseline vs year 2 and year 5) revealed significant changes in FA and Lt $(P<$ $.05)$ in the anterior aspect of the brain stem, genu and body of the corpus callosum, and bilateral corona radiata (Fig 2). In a post hoc analysis, results were significant for baseline vs year 2; however, no significant difference was observed between year 2 and year 5 .

Regarding L1 and MD, no significant difference was observed between patients and control participants at baseline. Repeatedmeasures ANOVA indicated that L1 did not significantly change during the 5-year observation period, whereas MD increased significantly in the genu and body of the corpus callosum and the bilateral corona radiata from baseline to 2 years (post hoc analysis 


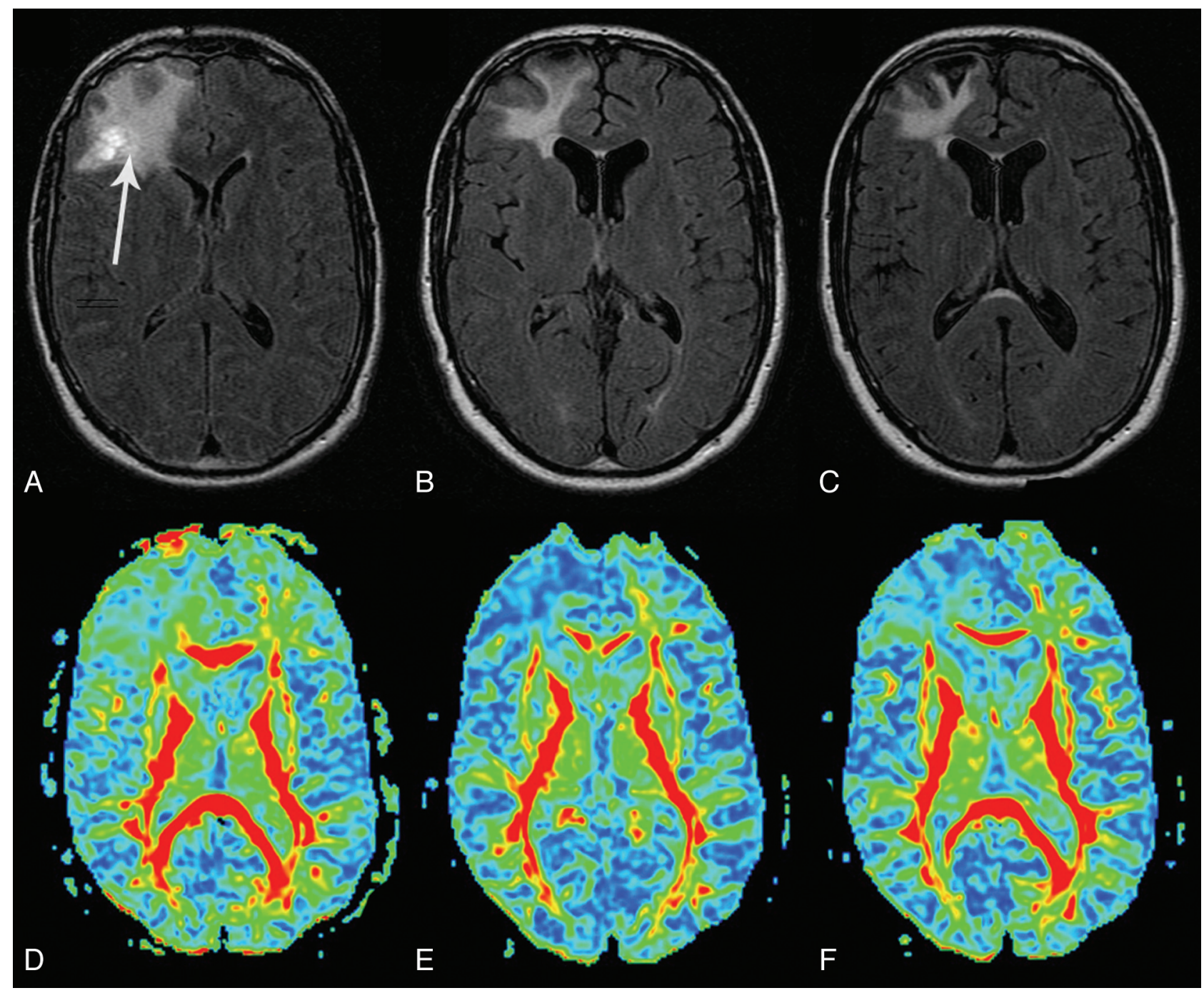

FIG 1. MR images of a patient with severe TBI at baseline $(A$ and $D)$, year 2 ( $B$ and $E)$, and year 5 ( $C$ and $F)$. On the conventional FLAIR images $(A-C)$, the hyperintensity of the right frontal lobe (arrow) decreases with time with some minimal atrophic changes. The corresponding FA maps ( $D-F)$ are presented. In the acute setting, the normalized FA was slightly reduced in the genu $(0.80)$ and body $(0.92)$ of the corpus callosum and in the bilateral corona radiata (right: 0.96; left: 0.98) compared with control participants (normalized value $=1$ ). At 2 years, most of the normalized FA values in these tracts had further decreased: genu (0.81) and body (0.79) of corpus callosum; corona radiata (right: 0.89 ; left: 0.96 ). At 5 years, the values slightly increased: genu (0.82) and body (0.85) of corpus callosum; corona radiata (right: 0.92; left: 0.97).

was significant for baseline vs year 2 but was not significant for year 2 vs year 5).

As presented in Fig 3, there were significant changes (year 2 vs baseline) in normalized FA (Fig 3A) and Lt (Fig 3B) at the anterior aspect of the brain stem, genu and body of the corpus callosum, and the right and left corona radiata in patients with TBI vs healthy control participants. At 5 years, neurocognitive sequelae were present in 5 patients (amnesia $[n=2]$, aphasia $[n=1]$, and dyspraxia $[n=2])$.

The baseline Lt and FA values at the anterior aspect of the brain stem, genu and body of the corpus callosum, and right and left corona radiata were significantly associated $(P<.05)$ with these neurocognitive sequelae at year 5 (including amnesia, aphasia, and dyspraxia) (Fig 4).

\section{DISCUSSION}

In this study, we found that FA in the genu and body of the corpus callosum and in the bilateral corona radiata were significantly lower in patients with severe TBI compared with control participants; these measures decreased significantly for the first 2 years after trauma and remained stable thereafter. The Lt in these same structures increased in the first 2 years and then remained stable.

These results indicate widespread white matter damage occurring in the setting of severe TBI and persisting with time, a finding that has been previously reported. ${ }^{14,26}$ The changes observed in FA were driven by alterations in radial (and not axial) diffusivity, suggesting that the underlying white matter alterations could be related to changes in myelin rather than axonal injury. ${ }^{6,27}$ The absence of changes for more than 2 years after TBI indicates that the most dynamic structural changes, and the window for therapeutic and rehabilitative intervention, may be limited beyond that time point. ${ }^{28}$

A pattern suggestive of myelin damage in white matter rather than axonal injury has been identified by other groups. ${ }^{8,14,26,29}$ Ewing-Cobbs et $\mathrm{al}^{8}$ evaluated 41 children at a 

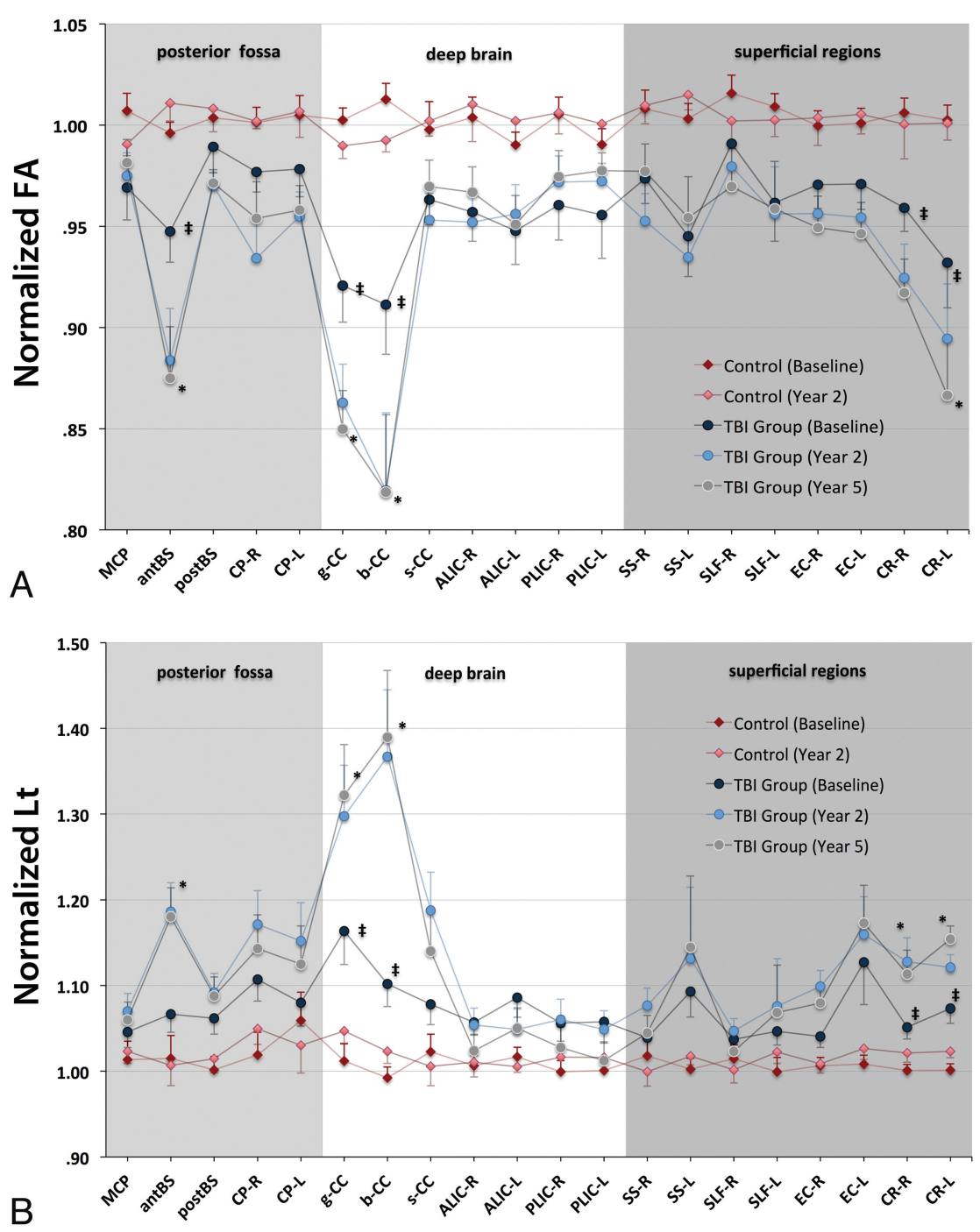

FIG 2. Normalized FA $(A)$, and $L t(B)$ values of patients and control participants at baseline and follow-up steps. The $x$-axis represents the ROIs (in posterior fossa: 1- MCP: middle cerebellar peduncle; 2- antBS: anterior brain stem; 3- postBS: posterior brain stem; 4- CP-R: right cerebral peduncle; 5- CP-L: left cerebral peduncle; in deep brain: 6- g-CC: genu of the corpus callosum; 7b-CC: body of the corpus callosum; 8-second-CC: splenium of the corpus callosum; 9- ALIC-R: right side anterior arm of the internal capsule; 10- ALIC-L: left side anterior arm of the internal capsule; 11- PLIC-R: right side posterior arm of the internal capsule; 12- PLIC-L: left side posterior arm of the internal capsule; in superficial brain regions: 13- SS-R: right stratum sagittale; 14- SS-L: left stratum sagittale; 15- SLF-R: right superior longitudinal fasciculus; 16- SLF-L: left superior longitudinal fasciculus; 17- EC-R: right external capsule; 18- EC-L: left external capsule; 19- CR-R: right corona radiata; $20-$ CR-L: left corona radiata). There were no significant differences in DTI parameters between baseline and year 2 in the control group and between year 2 and year 5 in the TBI group. 1: $P<.05$; for comparison of baseline values between control participants and patients (corrected $P$ ). *: $P<.05$; for repeated-measures ANOVA in the patient group between baseline and follow-up steps. Data are presented as mean and standard error of mean (handles). The connection lines are for clarity.

mean of 39 months after severe TBI, demonstrating (compared with a control group) significantly reduced corpus callosum FA and increased Lt, whereas L1 remained unchanged. This pattern was also seen in a recent study by Farbota et $\mathrm{al}^{29}$ on 12 patients with severe TBI. In a prospective cohort, Newcombe et $\mathrm{al}^{14}$ studied 68 patients who underwent MR imaging at a median of 11.8 months (range, 6.6 months to 3.7 years) after injury, finding significant decrements in FA in multiple structures including the corpus callosum, changes which were associated with increases in Lt and with clinical outcomes. Sidaros et $\mathrm{al}^{26}$ studied patients with severe TBI at 2 and 12 months after injury, demonstrating reductions in corpus callosum FA and increases in Lt. At 1 year, FA remained decreased and Lt increased particularly in patients with unfavorable outcome. Other authors have had slightly different results: In a study of 37 patients who underwent MR imaging with DTI at a mean of 8.9 years after TBI, Kraus et $\mathrm{al}^{6}$ found reduced $\mathrm{FA}$ in conjunction with increases in both Lt and L1 compared with matched control participants.

Our results also show that baseline FA and Lt measures of DTI (at the genu and body of the corpus callosum, anterior aspect of the brain stem, and right and left corona radiata) are associated with long-term cognitive sequelae in patients with severe TBI. Predicting neurobehavioral outcome in TBI is challenging because patients with similar clinical and radiologic characteristics in the acute phase of TBI may have markedly different outcomes ranging from death to complete recovery. ${ }^{2,13}$ In a prospective study, decreased regional FA in 4 major white matter tracts at 8 weeks was predictive of unfavorable outcome at 12 months. ${ }^{26}$ Available evidence indicates that damage to critical areas such as the corpus callosum or brain stem correlate most highly with poor post-TBI prognosis. ${ }^{26,28,30}$ In a large multicenter study, we recently generated and validated a stringent prognostic model by integrating a composite DTI score of white matter tracts with available clinical and CT-scan variables. Furthermore, DTI metric analysis has not only the potential to be applied clinically in an individual patient for prognosis assessment but also may be used in follow-up to monitor the response to therapeutic and rehabilitation measures.

Some limitations should be considered on interpretation of our results. TBI is biologically and clinically heterogeneous and may have a range of radiologic features. ${ }^{2}$ First, the results of this study could not be extrapolated to mild or moderate TBI, in which different temporal changes might occur. In addition, the heterogeneous spatial distribution of DTI and its various pathologic sequelae were a real challenge for ROI-based statistical analysis. In our study, the nonhomogeneous pattern of white matter injury was obvious in superficial brain regions, and 


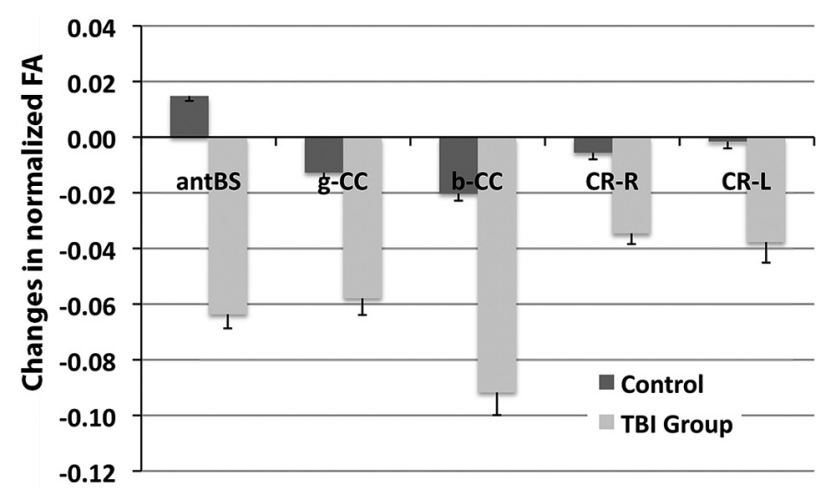

A

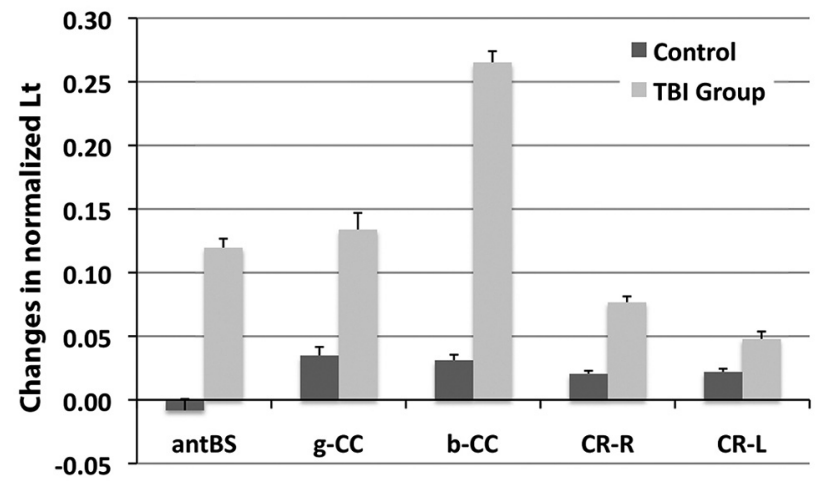

B

FIG 3. The changes (year 2 vs baseline) in normalized FA $(A)$ and Lt $(B)$ in the presented ROls were significantly higher in the patients vs healthy control participants. $P<.05$ for comparison between patients and control participants in all of the presented ROIs.

the high variability of DTI metrics observed in these regions was probably the result of the heterogeneous nature of the pathologic findings in TBI.

Furthermore, because we did not perform imaging between baseline and 2 years on these patients, we could not precisely identify at which time point the white matter stops evolving. Given the small patient sample and the heterogeneity of the conventional MR imaging findings, we did not analyze the conventional morphologic sequences. We reported the results of the DTI metrics only.

In an ideal setting, brain tissue diffusion measurements should be independent of the MR imaging scanner and image acquisition parameters. However, a recent study has indicated variations in apparent diffusion coefficients and FA values when images are acquired on different scanners or when different acquisition protocols are used. ${ }^{19}$ Therefore, we implemented a normalization step, which makes our estimates of DTI parameters comparable with results found in other groups despite slightly different MR imaging parameters. We believe that this approach helps to overcome data heterogeneity in quantitative DTI studies and can be an important step in clinical application of quantitative DTI measures. This study was designed and conducted based on a predetermined sample size of 15 patients to have a power $=80 \%$ and $\alpha=0.05$. Although we enrolled 15 patients in this study, 2 were dropped because of unwillingness to undergo the follow-up scan at 5 years. Therefore, larger long-term cohorts are needed to pro-
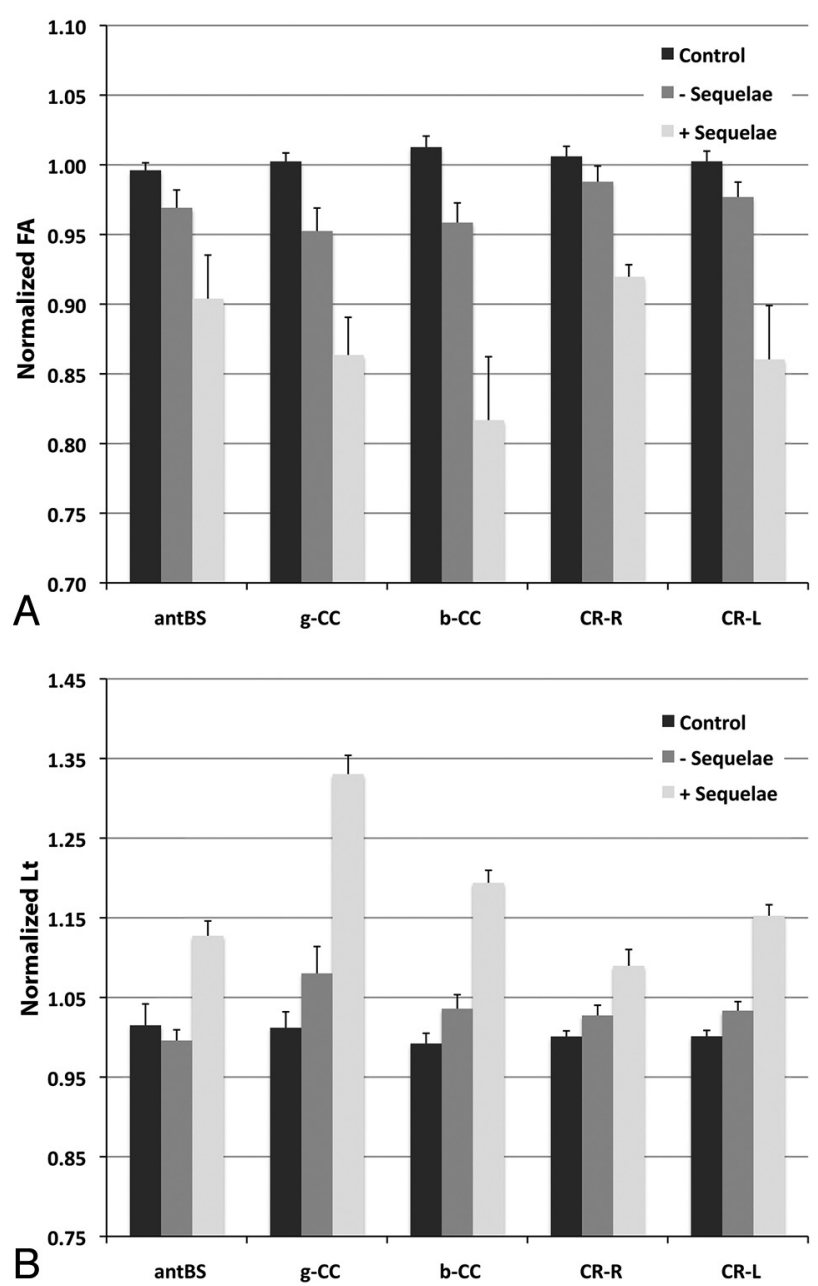

FIG 4. The normalized FA $(A)$ and $L t(B)$ values at baseline were significantly different in patients with cognitive sequelae at year 5 (including amnesia, aphasia, and dyspraxia) vs healthy control participants. $P<.05$ for ANOVA among the groups and for comparison between healthy control participants and patients with sequelae in all of the presented ROls.

vide a more comprehensive assessment and more thorough subgroup analysis.

\section{CONCLUSIONS}

This study demonstrates significant and durable changes in the corpus callosum and in the corona radiata in a cohort of patients with TBI who were observed during a 5 -year period. These alterations evolved significantly between the initial assessment and up to 2 years from the injury, beyond which few additional changes were seen. These data suggest that therapeutic and rehabilitative measures after severe TBI may be monitored by DTI during an initial time window, beyond which the microarchitecture of the white matter measured by TBI stop evolving.

Disclosures: Vincent Perlbarg—RELATED: Grant: Program "Investissements d'avenir" ANR-10-IAIHU-06; UNRELATED: Patents (planned, pending or issued): Patent Number FR 2975806 (A1). * Didier Dormont—RELATED: Grant: French Ministry of Health,* Comments: PHRC national (French Ministry of Health), Projet Hospitalier de Recherche Clinique \#P051061. Damien Galanaud-RELATED: French Ministry of Health,* Comments: Projet Hospitalier de Recherche Clinique \#P051061; UNRELATED: Consultancy: Olea Medical; Payment for Development of Educational Presentations: Sanofi Aventis; Travel/Accommodations/Meeting Expenses Unrelated to Activities 
Listed: Olea Medical. Louis Puybasset-RELATED: Grant: DRC, ${ }^{*}$ APHP, ${ }^{*}$ Comments: Funding by the French government, PHRC 2005 paid for the software used in the study; UNRELATED: Patents (planned, pending or issued): Patent published (no fees or money), Comments: http://patentscope.wipo.int/search/en/detail.jsf? docld =WO2012160316\&recNum =1\&maxRec $=$ \&office = \&prevFilter =\&sortOption = \&queryString $=\& \operatorname{tab}=\mathrm{PCT}+$ Biblio. *Money paid to institution

\section{REFERENCES}

1. Saatman KE, Duhaime AC, Bullock R, et al. Classification of traumatic brain injury for targeted therapies. I Neurotrauma 2008;25:719-38

2. Narayan RK, Michel ME, Ansell B, et al. Clinical trials in head injury. J Neurotrauma 2002;19:503-57

3. Smith DH, Meaney DF, Shull WH. Diffuse axonal injury in head trauma. J Head Trauma Rehabil 2003;18:307-16

4. Wada T, Asano Y, Shinoda J. Decreased fractional anisotropy evaluated using tract-based spatial statistics and correlated with cognitive dysfunction in patients with mild traumatic brain injury in the chronic stage. AJNR Am J Neuroradiol 2012;33:2117-22

5. Basser PJ, Mattiello J, LeBihan D. MR diffusion tensor spectroscopy and imaging. Biophys J 1994;66:259-67

6. Kraus MF, Susmaras T, Caughlin BP, et al. White matter integrity and cognition in chronic traumatic brain injury: a diffusion tensor imaging study. Brain 2007;130:2508-19

7. Kumar R, Husain M, Gupta RK, et al. Serial changes in the white matter diffusion tensor imaging metrics in moderate traumatic brain injury and correlation with neuro-cognitive function. $\mathrm{J} \mathrm{Neu}$ rotrauma 2009;26:481-95

8. Ewing-Cobbs L, Prasad MR, Swank P, et al. Arrested development and disrupted callosal microstructure following pediatric traumatic brain injury: relation to neurobehavioral outcomes. Neuroimage 2008;42:1305-15

9. Akpinar E, Koroglu M, Ptak T. Diffusion tensor MR imaging in pediatric head trauma. J Comput Assist Tomogr 2007;31:657-61

10. Wilde EA, Chu Z, Bigler ED, et al. Diffusion tensor imaging in the corpus callosum in children after moderate to severe traumatic brain injury. J Neurotrauma 2006;23:1412-26

11. Wilde EA, McCauley SR, Hunter JV, et al. Diffusion tensor imaging of acute mild traumatic brain injury in adolescents. Neurology 2008;70:948-55

12. Lipton ML, Kim N, Park YK, et al. Robust detection of traumatic axonal injury in individual mild traumatic brain injury patients: intersubject variation, change over time and bidirectional changes in anisotropy. Brain Imaging Behav 2012;6:329-42

13. Betz J, Zhuo J, Roy A, et al. Prognostic value of diffusion tensor imaging parameters in severe traumatic brain injury. $\mathrm{J} \mathrm{Neu}$ rotrauma 2012;29:1292-305
14. Newcombe V, Chatfield D, Outtrim J, et al. Mapping traumatic axonal injury using diffusion tensor imaging: correlations with functional outcome. PLoS One 2011;6:e19214

15. Yuan W, Holland SK, Schmithorst VJ, et al. Diffusion tensor MR imaging reveals persistent white matter alteration after traumatic brain injury experienced during early childhood. AJNR Am J Neuroradiol 2007;28:1919-25

16. Levin HS. Neuroplasticity following non-penetrating traumatic brain injury. Brain Inj 2003;17:665-74

17. Wu TC, Wilde EA, Bigler ED, et al. Longitudinal changes in the corpus callosum following pediatric traumatic brain injury. Dev Neurosci 2010;32:361-73

18. Young JA, Tolentino M. Neuroplasticity and its applications for rehabilitation. Am J Ther 2011;18:70-80

19. Galanaud D, Perlbarg V, Gupta R, et al. Assessment of white matter injury and outcome in severe brain trauma: a prospective multicenter cohort. Anesthesiology 2012;117:1300-10

20. Smith SM, Jenkinson M, Woolrich MW, et al. Advances in functional and structural MR image analysis and implementation as FSL. Neuroimage 2004;23 Suppl 1:S208-19

21. Pierpaoli C, Basser PJ. Toward a quantitative assessment of diffusion anisotropy. Magn Reson Med 1996;36:893-906

22. Smith SM, Jenkinson M, Johansen-Berg H, et al. Tract-based spatial statistics: voxelwise analysis of multi-subject diffusion data. Neuroimage 2006;31:1487-505

23. Mori S, Wakana S, Nagae-Poetscher L, et al. MRI Atlas of Human White Matter. Amsterdam: Elsevier; 2005

24. Luyt CE, Galanaud D, Perlbarg V, et al. Diffusion tensor imaging to predict long-term outcome after cardiac arrest: a bicentric pilot study. Anesthesiology 2012;117:1311-21

25. Benjamini Y, Hochberg Y. Controlling the false discovery rate: a practical and powerful approach to multiple testing. J Royal Stat Soc Ser B 1995;57:289-300

26. Sidaros A, Engberg AW, Sidaros K, et al. Diffusion tensor imaging during recovery from severe traumatic brain injury and relation to clinical outcome: a longitudinal study. Brain 2008;131:559-72

27. Mac Donald CL, Dikranian K, Bayly P, et al. Diffusion tensor imaging reliably detects experimental traumatic axonal injury and indicates approximate time of injury. J Neurosci 2007;27:11869-76

28. Huisman TA, Schwamm LH, Schaefer PW, et al. Diffusion tensor imaging as potential biomarker of white matter injury in diffuse axonal injury. AJNR Am J Neuroradiol 2004;25:370-76

29. Farbota KD, Bendlin BB, Alexander AL, et al. Longitudinal diffusion tensor imaging and neuropsychological correlates in traumatic brain injury patients. Front Hum Neurosci 2012;6:160

30. Parvizi J, Damasio AR. Neuroanatomical correlates of brainstem coma. Brain 2003;126:1524-36 